ISSN 0258-7122

Bangladesh J. Agril. Res. 33(3) : 479-492, September 2008

\title{
AGRO-MORPHOLOGICAL AND QUALITY CHARACTERIZATION OF BADSHAH BHOG GROUP FROM AROMATIC RICE GERMPLASM OF CHHATTISGARH
}

\begin{abstract}
RITA BISNE $^{1}$ AND A.K. SARAWGI ${ }^{2}$
India sub continent is a home for aromatic rice diversity. Land races and wild species possess immense potential of most valuable genes which can he effectively utilized in the present day breeding programmes to evolve miracle varieties in rice that possess not high yield potential and quality but also resistant to biotic and abiotic stresses. The present studies were carried out to characterize thirty two aromatic rice accessions of Badshah bhog group from IGKV, Raipur, Chhattisgarh germplasm. These germplasm accessions were evaluated for twenty-two morphological, six agronomical and eight quality characters. The specific genotypes B: 1340, B: 2039, B: 2495, B: 2816, B: 16930 B: 2354, B:1639, B:2094 were identified for quality and agronomical characteristics. These may be used in hybridization programme to achieve desired segregants for good grain quality with higher yield.
\end{abstract}

Key Words: Morphological characters. quality characters, characterization in aromatic rice.

\section{Introduction}

The cultivated rice of Asia (Oryza sativa L.) is supposed to have originated in the South and/ or South East Asia. India forms a major part of this region Thus, it is traditionally rich in the diversity of rice including the wild progenitors of cultivated rice (Singh et al., 2001). Genetic diversity probably serves as an insurance against crop failure (Subba Rao et al., 2001). Landraces and wild species possess immense potential of most valuable genes which can be effectively utilized in the present day breeding programmes to evolve miracle varieties in rice that possess not high yield potential and quality, but also resistant to biotic and abiotic stresses (Saxena et al., 1988). Collection and characterization of this germplasm is not only important for utilizing the appropriate attribute based donors in breeding programmes, but is also essential in the present era for protecting the unique rice.

Indian sub continent is a home for aromatic rice diversity. Aroma quality of scented rice is a major character which increases the value of rice in international market (Nayak et al., 2002). The demand for special purpose aromatic rice has dramatically increased over the past two decades. India emerges as one of the

${ }_{1 \& 2}$ Department of Plant Breeding and Genetics, College of Agriculture, Indira Gandhi Krishi Vishwavidyalaya, Raipur, C.G -492006, India. 
major exporter of rice in international markets. The success of any breeding programme depends upon the selection of the parents for hybridization.

\section{Materials and Method}

Collection and characterization of existing germplasm is not only important for utilizing the appropriate attribute based donors in breeding programmes, but is also essential in the present era for protecting the unique rice. A total of 32 aromatic rice accessions were selected from a collection of aromatic rice germplasm in IGKV, Raipur on the basis of their nomenclature. Morphological descriptors are presented in Table 1 . Twenty five days old seedlings were transplanted with a spacing of $20 \mathrm{~cm}$ and $15 \mathrm{~cm}$ between rows and between plants, respectively. Observations were recorded on five randomly selected plants for 22 morphological descriptors viz., 1. Leaf Length (LL) in $\mathrm{cm}$ at heading stage 2. Leaf Width (LW) in $\mathrm{cm}$ at heading stage, 3. Leaf Blade Pubscence (LBP) at booting and heading stage with classes 1 . Glabrous, 2. Intermediate, 3. Pubscent 4. Leaf Blade Colour (LBC) at stem elongation to heading stage with classes 1. Light green, 2. Green, 3. Drak green, 4. Purple tips, 5. Purple margin. 6. Purple blotch, 7. Purple; 5. Basal Leaf Sheath Colour (BLSC) at early to late vegetative stage with classes 1. Green, 2. Purple lines 3. Light purple, 4. Purple; 6. Flag Leaf Angle (FLA) at stem elongation to booting stage with classes 1. Erect, 3. Intermediate, 5. Horizontal, 6. Descending (more than 90 degree), 7. Ligule Colour (LC) at stem elongation to booting stage with classes 1 . White, 2. Purple lines, 3. Purple. 8. Collor Colour (CC) at stem elongation to booting stage with classes 1. Light green, 2. Green, 3. Purple, 9. Auricle Colour (AC) classes 1. Light green, 2. Purple; 10. Culm Angle (CmA) at milk stage to maturity stage with classes 1. Erect, 3. Intermediate, 5. Open, 7. Spreading. 9. Procumbent; 11. Panicle Type (PnT) at dough stage with classes 1. Compact, 5. Intermediate, 9 Open, 12. Secondary Branching of Panicles $(\mathrm{PnBr})$ at dough stage classes 0 . Absent, 1. Light, 2 Heavy, 3. Clustery, 13 Panicle Axis (PnAK) at milk stage to mature stage with classes 1 . Straight, 2. Droopy; 14 Panicle Exertion (ExS) at milk stage to mature stage with classes 1 . Well exerted, 2. Moderately well exerted, 3 Just exerted, 7. Partly exerted, 9. Enclosed; 15. Panicle Threshability (Thr) at mature stage with classes 1 . Difficult $(>1 \%)$, 3. Moderately diftcu1t (l5\%), 5. Intermediate (6-25\%), 7. Loose (26-50\%), 9. Easy (5l-100\%); 16. Awning (An) at milk stage to mature stage with classes 0. Absent, 1. Short and partly awned, 5. Short and fully awned; 7. Long and party awned, 9. Long and fully awned; 17 Awn colour (AnC) at heading stage with classes 0 . Awnless, 1. Straw, 2. Gold, 3. Brown (Twany), 4. Red, 5. Purple, 6. B1ack; 15. Apiculus 
Colour (ApC) at milk stage to mature stage with classes 1. White, 2. Straw, 3 Brown. 4. Red, 5. Red Apex, 6. Purple, 7. Purple Apex; 19. Stigma Colour (SgC) at heading stage with classes 1 . White, 2. Light green, 3. Yellow, 4. Light purple, 5. Purple; 20. Lemma and Palea Colour (LmPC) at mature stage with classes 0 straw, 1. Gold and gold furrow on straw background, 2. Brown furrow on straw, 4. Brown (Twany), 5. Reddish to light purple, 6. Purple spots on straw, 7. Purple furrow on straw, 8 Purple, 9. Black, 10. White; 21. Lemma and Palea Pubscence $(\mathrm{LmPb})$ at mature stage with classes 1. Glabrous, 2. Hairs on lemma keel, 3. Hairs on upper portion, 4. Short hairs, 5. Long hairs (Velvety); 22. Sterile Lemma Colour (SLmc) at mature stage with classes 1. Straw (Yellow), 2. Gold, 3. Red, 4. Purple. Six agronomical and yield traits were recorded, such as 1 . Days to 50\% flowering (DF50\%), 2. Plant Height (PH) in cm, 3. Panicle Length (PL) in cm, 4. Total tillers per hill (TT/hill), 5. Effective tillers per hill (ET/hill) and 6. Grain Yield (GY) in kg/ha. Eight quality traits were also recorded i.e., 1. Hulling percentage (Hull \%), 2. Milling percentage (Mill \%), 3. Head Rice Recovery (HRR\%), 4. Kernel Length (L) in mm, 5. Kernel Breadth (B) in mm, 6. Length Breadth ratio (L/B ratio), 7. Alkali Spreading Value (ASV), and 8. Aroma.

\section{Results and Discussion}

\section{A. Morphological characters}

Twenty two morphological characters were recorded for 32 accessions. The morphological characters included leaf length (1), leaf width (2), leaf blade pubescence (3), leaf blade colour (4), basal leaf sheath colour (5), flag leaf angle (6) ligule colour (7), collor colour (8), auricle colour (9) culm angle (10), panicle type (11), secondary branching of panicles (12), panicle axis (13), panicle exertion (14), panicle threshability (15), awning (16), awn colour (17), apiculus colour (18), stigma colour (19), lemma and palea colour (20), lemma and palea pubescence (21), and sterile lemma colour (22). The results of morphological characters recorded for 32 accessions are discussed in Table 1 (Character 1 to 22) and its graphical representation of frequency distribution showed in Fig 1. Out of 22 morphological characters, leaf blade colour (4) and lemma and palea colour (20), apiculus colour (18) and Lemma and Palea Pubescnece (21) found highest variation in different accessions. After that, leaf Blade Pubscence (1), flag Leaf Angle (6) culm angle (10), panicle type (11), panicle threshability (15) showed variation in different accessions and rest 7 found each of two classes among different accessions (Fig. 1 and Table 1). The remaining 6 morphological characters showed no difference among accessions. 
Fig 1. Frequency Distribution of morphological characters of Badshah Bhog Group.
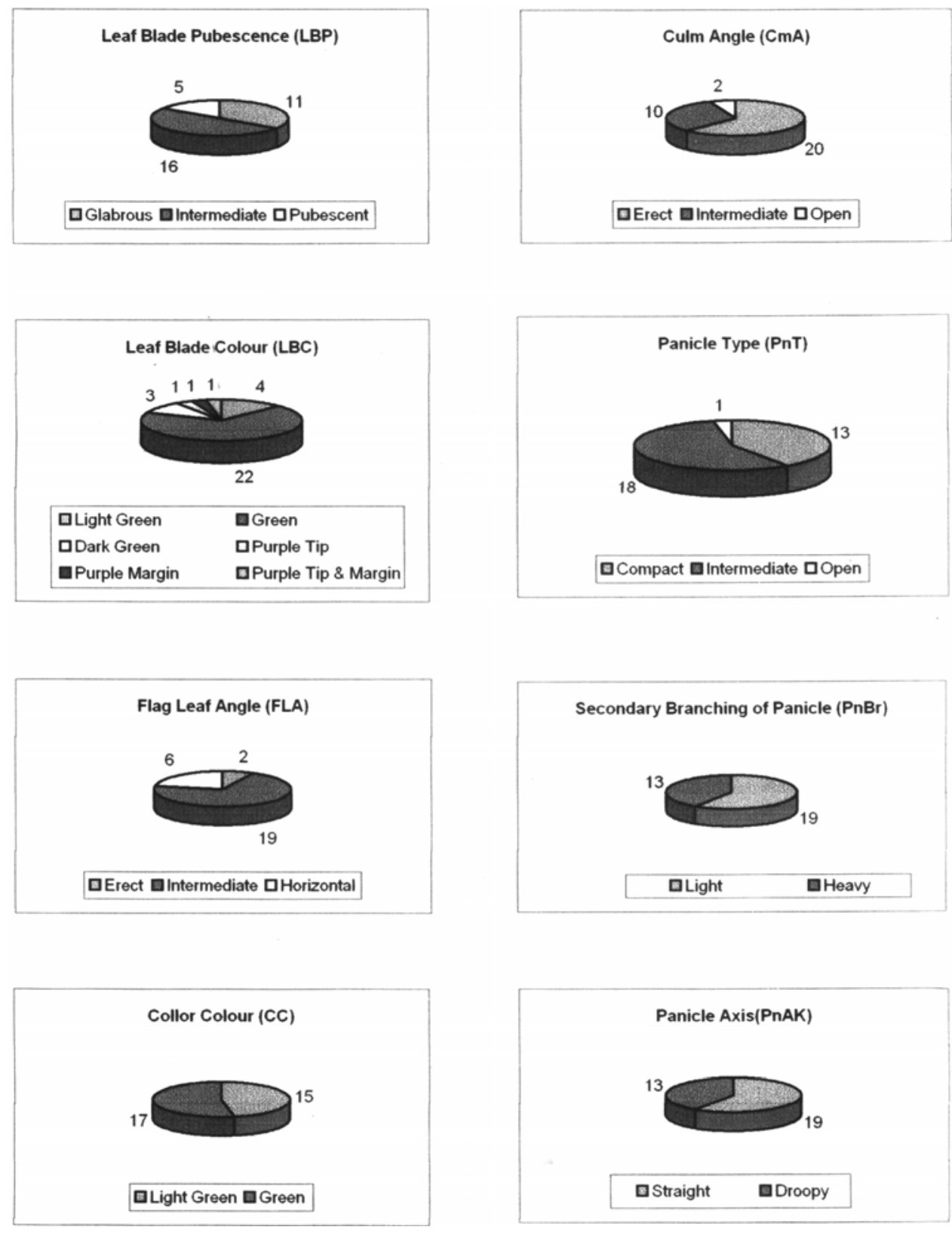
AGRO-MORPHOLOGICAL AND QUALITY CHARACTERIZATION

483

Cont'd. Fig 1.
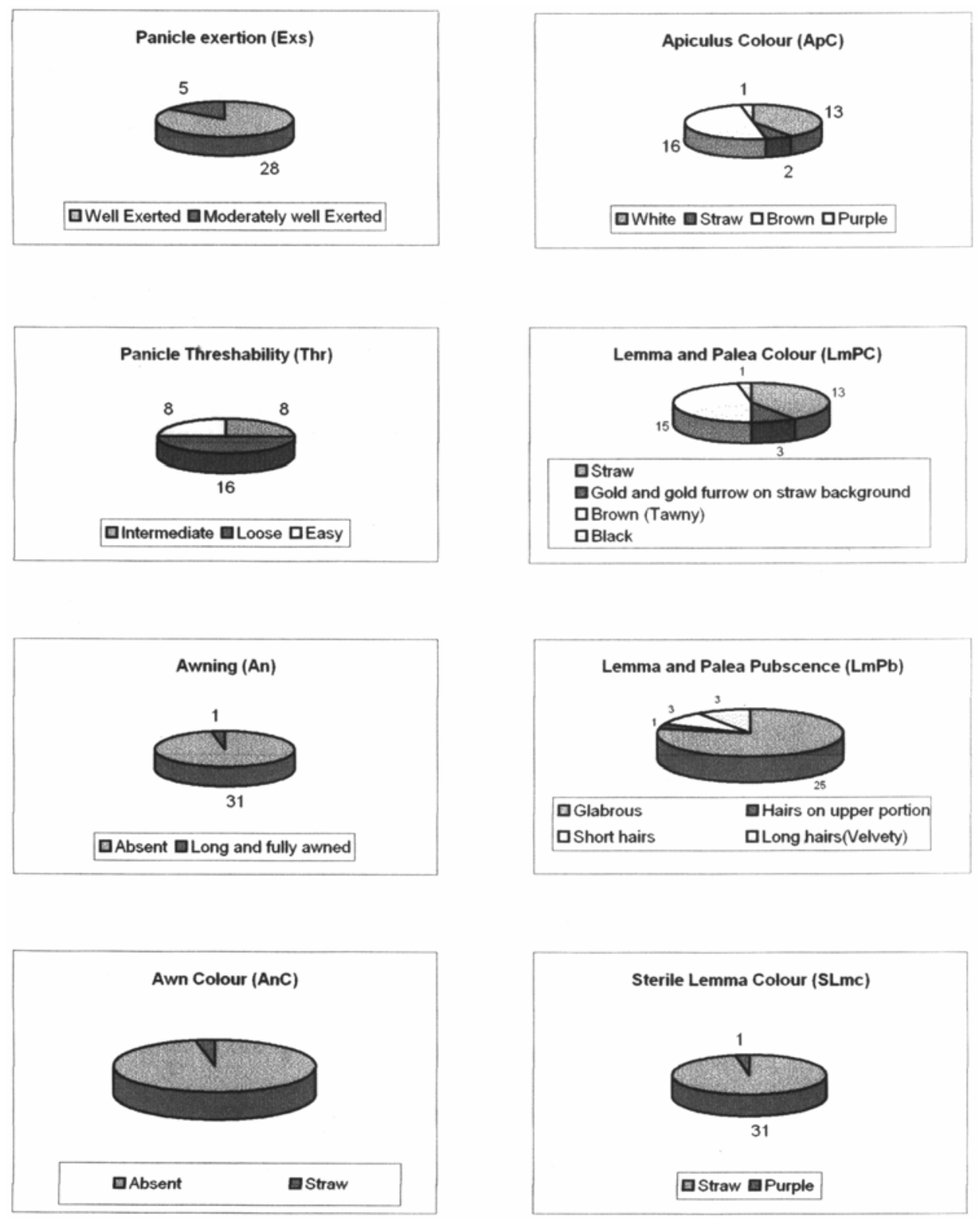
Table 1. Morphological traits of Badshah Bhog Group from aromatic rice germplasm.

\begin{tabular}{|c|c|c|c|c|c|c|c|c|c|c|c|c|c|c|c|c|c|c|c|c|c|c|c|}
\hline ACC No. & Designation & 1 & 2 & 3 & 4 & 5 & 6 & 7 & 8 & 9 & 10 & 11 & 12 & 13 & 14 & 15 & 16 & 17 & 18 & 19 & 20 & 21 & 22 \\
\hline B: 1209 & Basabhog & 61.2 & 1.1 & 2 & 2 & 1 & 3 & 1 & 2 & 1 & 1 & 5 & 1 & 1 & 1 & 5 & 0 & 0 & 1 & 1 & 0 & 1 & 1 \\
\hline B: 421 & Badshah Bhog & 54.5 & 1.1 & 2 & 5 & 1 & 6 & 1 & 1 & 1 & 3 & 5 & 1 & 2 & 1 & 5 & 0 & 0 & 6 & 1 & 9 & 1 & 4 \\
\hline B: 54 II & Badshah Bhog & 58.8 & 0.9 & 1 & 3 & 1 & 3 & 1 & 1 & 1 & 3 & 5 & 1 & 1 & 1 & 5 & 0 & 0 & 3 & 1 & 4 & 1 & 1 \\
\hline B: 232 I & Badshah Bhog & 43.5 & 1.4 & 2 & 2 & 1 & 3 & 1 & 2 & 1 & & 5 & 1 & 1 & 1 & 5 & 0 & 0 & 3 & 1 & 4 & 1 & 1 \\
\hline B: 323 II & Badshah Bhog & 68.4 & 1.3 & 3 & 2 & 1 & 5 & 1 & 2 & 1 & 1 & 5 & 1 & 1 & 1 & 5 & 0 & 0 & 1 & 1 & 0 & 4 & 1 \\
\hline B: 528 & Badshah Bhog & 57.2 & 1.1 & 1 & 2 & 1 & 3 & 1 & 2 & 1 & 1 & 5 & 1 & 1 & 1 & 5 & 0 & 0 & 3 & 1 & 4 & 1 & 1 \\
\hline B:799 II & Badshah Bhog & 60.2 & 1.1 & 1 & 1 & 1 & 5 & 1 & 2 & 1 & 3 & 5 & 1 & 1 & 1 & 5 & 0 & 0 & 3 & 1 & 4 & 1 & 1 \\
\hline B: 1005 & Badshah Bhog & 46.6 & 0.9 & 2 & 2 & 1 & 3 & 1 & 1 & 1 & 1 & 5 & 1 & 2 & 1 & 5 & 0 & 0 & 3 & 1 & 4 & 1 & 1 \\
\hline B: 1029 & Badshah Bhog & 66.3 & 1.2 & 2 & 4,5 & 1 & 6 & 1 & 2 & 1 & 3 & 1 & 2 & 1 & 2 & 5 & 0 & 0 & 1 & 1 & 0 & 1 & 1 \\
\hline B: 1307 & Badshah Bhog & 32.8 & 1.2 & 1 & 2 & 1 & 1 & 1 & 1 & 1 & 5 & 5 & 1 & 2 & 1 & 5 & 0 & 0 & 1 & 1 & 0 & 5 & 1 \\
\hline B: 1322 & Badshah Bhog & 54.2 & 1.1 & 1 & 1 & 1 & 6 & 1 & 1 & 1 & 3 & 1 & 2 & 1 & 1 & 5 & 0 & 0 & 3 & 1 & 4 & 1 & 1 \\
\hline B:1340 & Badshah Bhog & 43.5 & 0.9 & 2 & 2 & 1 & 3 & 1 & 1 & 1 & 1 & 1 & 1 & 2 & 1 & 5 & 0 & 0 & 1 & 1 & 4 & 1 & 1 \\
\hline B: 1389 & Badshah Bhog & 48.9 & 0.9 & 1 & 2 & 1 & 3 & 1 & 1 & 1 & 1 & 5 & 1 & 1 & 2 & 5 & 0 & 0 & 1 & 1 & 0 & 1 & 1 \\
\hline B: 1693 & Badshah Bhog & 43.5 & 0.8 & 1 & 2 & 1 & 3 & 1 & 2 & 1 & 3 & 1 & 2 & 2 & 1 & 5 & 0 & 0 & 3 & 1 & 4 & 1 & 1 \\
\hline B.1727 & Badshah Bhog & 38.9 & 0.9 & 1 & 2 & 1 & 3 & 1 & 2 & 1 & 1 & 1 & 2 & 1 & 1 & 5 & 0 & 0 & 3 & 1 & 4 & 4 & 1 \\
\hline B. 2094 & Badshah Bhog & 40.1 & 1.1 & 3 & 1 & 1 & 3 & 1 & 2 & 1 & 1 & 5 & 1 & 1 & 1 & 5 & 0 & 0 & 2 & 1 & 4 & 1 & 1 \\
\hline B: 2354 & Badshah Bhog & 61.9 & 1.3 & 2 & 2 & 1 & 3 & 1 & 2 & 1 & 1 & 1 & 2 & 1 & 1 & 5 & 0 & 0 & 1 & 1 & 0 & 1 & 1 \\
\hline B: 2461 & Badshah Bhog & 71.5 & 0.9 & 3 & 2 & 1 & 3 & 1 & 2 & 1 & 1 & 1 & 1 & 2 & 1 & 3 & 0 & 0 & 1 & 1 & 0 & 1 & 1 \\
\hline B: 2495 & Badshah Bhog & 56.3 & 1.2 & 1 & 2 & 1 & 3 & 1 & 1 & 1 & 3 & 1 & 2 & 1 & 1 & 3 & 0 & 0 & 3 & 1 & 4 & 1 & 1 \\
\hline B: 2504 & Badshah Bhog & 59.8 & 1.1 & 2 & 2 & 1 & 1 & 1 & 1 & 1 & 1 & 1 & 1 & 1 & 2 & 3 & 0 & 0 & 1 & 1 & 1 & 1 & 1 \\
\hline B: 2812 & Badshah Bhog & 50.5 & 1.2 & 2 & 2 & 1 & 3 & 1 & 2 & 1 & 1 & 5 & 1 & 1 & 1 & 7 & 0 & 0 & 3 & 1 & 4 & 1 & 1 \\
\hline
\end{tabular}




\section{Table 1. Cont'd}

\begin{tabular}{|c|c|c|c|c|c|c|c|c|c|c|c|c|c|c|c|c|c|c|c|c|c|c|c|}
\hline B. 2814 & Badshah Bhog & 54.9 & 0.8 & 1 & 2 & 1 & 1 & 1 & 1 & 1 & 1 & 1 & 2 & 1 & 2 & 7 & 0 & 0 & 1 & 1 & 0 & 1 & 1 \\
\hline B: 1717 & Badshah Bhog & 59.3 & 0.9 & 2 & 1 & 1 & 3 & 1 & 1 & 1 & 1 & 1 & 2 & 1 & 1 & 7 & 0 & 0 & 1 & 1 & 0 & 5 & 1 \\
\hline B: 2604 & Badshah Bhog & 63.8 & 1.1 & 2 & 2 & 1 & 6 & 1 & 2 & 1 & 1 & 1 & 2 & 1 & 1 & 7 & 0 & 0 & 3 & 1 & 0 & 1 & 1 \\
\hline B: 2464 & Badshah Bhog & 57.2 & 0.9 & 2 & 3 & 1 & 5 & 1 & 1 & 1 & 3 & 9 & 1 & 2 & 1 & 3 & 0 & 0 & 3 & 1 & 4 & 1 & 1 \\
\hline B: 6701 & Badshah Bhog & 57.5 & 1.2 & 2 & 2 & 1 & 5 & 1 & 1 & 1 & 1 & 5 & 2 & 2 & 1 & 3 & 0 & 0 & 3 & 1 & 1 & 1 & 1 \\
\hline B: 1713 & Badshah Bhog & 44.5 & 1.3 & 3 & 2 & 1 & 3 & 1 & 1 & 1 & 5 & 5 & 1 & 2 & 1 & 3 & 0 & 0 & 3 & 1 & 4 & 3 & 1 \\
\hline B: 2039 & Badshah Bhog & 52.6 & 1.3 & 2 & 3 & 1 & 5 & 1 & 2 & 1 & 3 & 5 & 2 & 2 & 1 & 7 & 0 & 0 & 2 & 1 & 0 & 5 & 1 \\
\hline B: 2816 & Badshah Bhog & 70.6 & 1.1 & 2 & 2 & 1 & 3 & 1 & 2 & 1 & 1 & 1 & 2 & 1 & 2 & 7 & 9 & 1 & 1 & 1 & 0 & 1 & 1 \\
\hline Bd: 105 & Badshah Bhog & 52.1 & 1.1 & 2 & 4 & 1 & 5 & 1 & 1 & 1 & 3 & 5 & 2 & 2 & 1 & 7 & 0 & 0 & 1 & 1 & 0 & 1 & 1 \\
\hline
\end{tabular}




\section{B. Agronomical characters}

Thirty two accessions of Badshahbhog group were evaluated for agronomical traits like plant height, panicle length, total tillers per hill and effective tillers per hill were recorded from five competitive plants of middle row of each entry, while days to $50 \%$ flowering and grain yield were on the plot basis.

Table 2. Mean performance of agronomical characteristics of Badshah Bhog group.

\begin{tabular}{|c|c|c|c|c|c|c|c|c|}
\hline Designation & & $\begin{array}{c}\mathrm{DF} \\
(50 \%)\end{array}$ & $\mathrm{PH}(\mathrm{cm})$ & $\mathrm{PL}(\mathrm{cm})$ & TT/hill & ET/hill & $\begin{array}{c}\mathrm{GY} \\
(\mathrm{kg} / \mathrm{ha})\end{array}$ & Rank \\
\hline Badshah Bhog & B: 1209 & 107 & 155 & 27 & 72 & 5.8 & 2889 & 18 \\
\hline Badshah Bhog & B: 42 & 94 & 160.2 & 24.4 & 5.8 & 4.4 & 1833 & 29 \\
\hline Badshah Bhog & B:54 II & 101 & 146 & 254 & 70 & 4.0 & 1546 & 31 \\
\hline Badshah Bhog & B: 232 I & 102 & 164.4 & 27 & 86 & 7.2 & 2833 & 22 \\
\hline Badshah Bhog & B: 323 II & 108 & 146 & 25.8 & 8.4 & 5.4 & 2620 & 26 \\
\hline Badshah Bhog & B: 528 & 106 & 156 & 26 & 8.2 & 6.2 & 2667 & 24 \\
\hline Badshah Bhog & B: 799 II & 104 & 164 & 264 & 74 & 64 & 1380 & 32 \\
\hline Badshah Bhog & B: 1005 & 105 & 1558 & 26 & 96 & 74 & 2898 & 17 \\
\hline Badshah Bhog & B:1029 & 104 & 153.4 & 27 & 70 & 5.6 & 2204 & 28 \\
\hline Badshah Bhog & B: 1307 & 78 & 105.6 & 27.4 & 58 & 5.0 & 1667 & 30 \\
\hline Badshah Bhog & B: 1322 & 100 & 154.8 & 27 & 74 & 52 & 2648 & 25 \\
\hline Badshah Bhog & B: 1340 & 99 & 135.6 & 26.2 & 7.0 & 6.4 & 3194 & 10 \\
\hline Badshah Bhog & B: 1389 & 108 & 145.6 & 274 & 72 & 64 & 3130 & 11 \\
\hline Badshah Bhog & B: 1693 & 103 & 156.2 & 27.6 & 84 & 70 & 3231 & 9 \\
\hline Badshah Bhog & B: 1727 & 104 & 155,6 & 25.4 & 9.4 & 6.2 & 3296 & 8 \\
\hline Badshah Bhog & B:2094 & 100 & 155.2 & 26.8 & 7.0 & 5.6 & 3685 & 5 \\
\hline Badshah Bhog & B:2354 & 105 & 157 & 28 & 8.2 & 5.8 & 4056 & 3 \\
\hline Badshah Bhog & B: 2461 & 103 & 1678 & 27.4 & 76 & 5.6 & 3361 & 6 \\
\hline Badshah Bhog & B: 2495 & 109 & 174 & 304 & 76 & 5.6 & 3361 & 7 \\
\hline Badshah Bhog & B: 2504 & 109 & 156.6 & 27.6 & 6.8 & 6.0 & 3130 & 12 \\
\hline Badshah Bhog & B:2812 & 103 & 156.6 & 27.8 & 9.0 & 7.2 & 2843 & 21 \\
\hline Badshah Bhog & B: 2814 & 109 & 166.4 & 286 & 8.2 & 6.0 & 3102 & 14 \\
\hline Badshah Bhog & B: 1717 & 109 & 145 & 27 & 7.8 & 7.8 & 2852 & 20 \\
\hline Badshah Bhog & B: 2604 & 107 & 1548 & 26.6 & 90 & 5.4 & 3111 & 13 \\
\hline Badshah Bhog & B: 2302 & 99 & 154.4 & 25.6 & 7.2 & 6.4 & 2991 & 15 \\
\hline Badshah Bhog & B:2464 & 102 & 1662 & 28.2 & 8.8 & 6.4 & 2963 & 16 \\
\hline Badshah Bhog & B: 670 I & 91 & 162.8 & 28 & 78 & 7.0 & 2583 & 27 \\
\hline Badshah Bhog & B: 1639 & 99 & 180 & 29.2 & 10.6 & 6.2 & 3722 & 4 \\
\hline Badshah Bhog & B: 1713 & 102 & 179.4 & 24.8 & 8.2 & 6.2 & 2741 & 23 \\
\hline Badshah Bhog & B: 2039 & 104 & 170.2 & 30.6 & 78 & 6.6 & 4389 & 2 \\
\hline Badshah Bhog & B: 2816 & 103 & 146.6 & 23.4 & 78 & 7.4 & 4630 & 1 \\
\hline Badshah Bhog & Bd: 105 & 104 & 157.2 & 26.4 & 126 & 6.4 & 2870 & 19 \\
\hline \multicolumn{9}{|l|}{ Range } \\
\hline Min & & 78 & 105.6 & 23.4 & 5.8 & 40 & 1380 & \\
\hline Max & & 109 & 180.0 & 306 & 12.6 & 7.8 & 4630 & \\
\hline
\end{tabular}

DF: Days to 50\% flowering, PH:Plant Height, PL: Panicle length.TT/hill: Total tillers per hill ET/hill: Effective tillers per hill, GY (kg/ha): Gram yield (kg/ha). 
Of the 32 accessions, 10 top ranking accessions were identified based on the mean yield performance during 2003 and 2004. The highest grain yield (4630 $\mathrm{kg} / \mathrm{ha}$ ) was found in accession B: 2816 followed by B: 2039, B: 2354. B: 1639, B: 2094, B: 2461, B: 2495, B: 1727, B: 1693 and B: 1340 (Table 2). Out of 32, only 14 accessions showed more than $3000 \mathrm{~kg} / \mathrm{ha}$ grain yield. Grain yield ranged between $1380 \mathrm{~kg} / \mathrm{ha}$ (B: 79911) to $4630 \mathrm{~kg} / \mathrm{ha} \mathrm{(B:} \mathrm{2816).}$

The character days to $50 \%$ flowering ranged from 78 days (B: 1307) to 109 Days (B: 2495, B: 2504, B: 2814 and B: 1717) and plant height ranged from 105.6 cm (B: 1307) to $180 \mathrm{~cm}$ (B: 1639). The accessions B: 2039, B: 2495, B: 1639, B: 2814, B: 2464, B: 2354, B: 6701 were found to have longer panicle length and it ranged between $4 \mathrm{~cm}$ (B: 2816) and $30.6 \mathrm{~cm}$ (B: 2039). For the character total tillers per hill, more number of tillers (more than or equal to 9 ) were found in accessions Bd: 105, B: 1639, B: 1005, B: 1727, B: 2812, B: 2604 and it ranged between Bd: 105 (12.6) to B: 42 I(5.8). The character effective tillers per hill ranges between Bd: 105 (9.4) to B: 54 11(4.0) and found higher number (more than 7) of effective tillers were found in accessions B: 1717, B: 1005, B: 2816, B: 2812, B: 2321 (Table 2).

\section{Quality characters}

Quality characteristics of the rice grain are related to a complex physico-chemical property. Some have excellent kernel shape and kernel length/breadth ratio, which are the important features of quality rices (Patil et al., 2003). For the character hulling percentage, all the accessions were found to have more than 80 percent hulling value except three viz., B: $421 \mathrm{Bd}: 105,8.1307$. The character milling percentage more than 75 percent is desirable for suitable screening of genotypes found in 14 accessions B: 1029, B: 79911, B: 1322,B:2354.B6701.B 1717,8 1693,8 246l,B 246411421 B: 528, B: 2504, B: 1639, B: 2039. For quality evaluation, head rice recovery is one ol the most important characters, so more than 65 percent head rice recovery percentage is desirable, which was found in 6 accessions B: 1029, B:799 11, B: 1389, B 528, B: 249, B: 2604 (Table 3).

For the character kernel length (mm) 2 accessions viz. B: 1307, B: 2816 were found to have more than $3 \mathrm{~mm}$ length of kernel and in length breadth ratio accession were found to have more than 3 of $\mathrm{L} / \mathrm{B}$ ratio. According to grain dimension of milled rice, out of 32 evaluated for shape and size depending upon kernel length and length/breadth ratio, the accession B:1389 is short-slender, B: 2816, B: 1307 long slender 26 in short medium and remaining 9 accessions were short bold type according to International rice shape and size from IRRI (Table 3 and 4). 
Table 3. Grain quality characteristics of Badshah Bhog group.

\begin{tabular}{|c|c|c|c|c|c|c|c|c|c|c|}
\hline Entry No & ACC. No. & Designation & HULL( \%) & MILL (\%) & HRR (\%) & $\mathrm{KL}(\mathrm{mm})$ & $\mathrm{KB}(\mathrm{mm})$ & $\mathrm{L} / \mathrm{B}$ & A.S.V. & AROMA \\
\hline 54 & B: 1209 & Basabhog & 80.74 & 74.99 & 57.81 & 4.3 & 2.1 & 2.05 & 6.00 & MS \\
\hline 56 & B: 54 II & Badshah Shog & 81.75 & 72.50 & 53.00 & 4.1 & 1.9 & 2.16 & 5.83 & SS \\
\hline 58 & B: 323 II & Badshah Shog & 82.13 & 72.08 & 64.74 & 4.4 & 2.0 & 2.20 & 5.67 & SS \\
\hline 59 & B: 528 & Badshah Shog & 81.72 & 75.56 & 65.53 & 4.0 & 2.0 & 2.00 & 6.00 & SS \\
\hline 60 & B:799 II & Badshah Shog & 82.10 & 78.39 & 66.11 & 4.4 & 2.1 & 2.10 & 5.33 & S \\
\hline 63 & B: 130 & Badshah Shog & 79.33 & 72.69 & 39.02 & 6.8 & 2.1 & 3.24 & 4.33 & S \\
\hline 64 & B: 1322 & Badshah Shog & 81.99 & 78.22 & 60.79 & 4.0 & 2.1 & 1.90 & 6.00 & MS \\
\hline 65 & B: 1340 & Badshah Shog & 81.16 & 73.64 & 44.10 & 4.0 & 2.1 & 1.90 & 6.00 & SS \\
\hline 66 & B:1389 & Badshah Shog & 81.73 & 73.68 & 65.69 & 3.9 & 1.2 & 3.25 & 5.83 & SS \\
\hline 67 & B: 1693 & Badshah Shog & 81.31 & 75.77 & 63.95 & 3.7 & 2.0 & 1.85 & 6.00 & SS \\
\hline 72 & B: 2495 & Badshah Shog & 80.98 & 73.77 & 65.26 & 4.1 & 2.1 & 1.95 & 6.00 & SS \\
\hline 73 & B: 2504 & Badshah Shog & 81.Q3 & 75.24 & 60.02 & 4.2 & 2.0 & 2.10 & 6.33 & MS \\
\hline 74 & B: 2812 & Badshah Shog & 81.22 & 74.23 & 57.33 & 4.2 & 1.9 & 2.21 & 6.33 & MS \\
\hline 75 & B:2814 & Badshah Shog & 81.85 & 72.25 & 58.89 & 4.1 & 2.0 & 2.05 & 6.00 & SS \\
\hline 76 & B: 1717 & Badshah Shog & 82.31 & 76.65 & 62.63 & 4.1 & 2.1 & 1.95 & 5.67 & SS \\
\hline 77 & B: 2604 & Badshah Shog & 81.96 & 74.40 & 65.25 & 4.1 & 2.1 & 1.95 & 4.00 & SS \\
\hline 192 & B: 2302 & Badshah Shog & 81.26 & 74.27 & 61.65 & 4.0 & 2.0 & 2.00 & 4.83 & MS \\
\hline 193 & B: 2464 & Badshah Shog & 80.58 & 75.57 & 57.78 & 4.1 & 1.9 & 2.16 & 5.50 & SS \\
\hline
\end{tabular}


Table 3. Cont'd

\begin{tabular}{lllllllllll}
\hline 214 & B: 670$)$ & Badshah Shog & 81.90 & 78.00 & 50.38 & 4.1 & 2.2 & 1.86 & 5.67 & MS \\
215 & B: 1639 & Badshah Shog & 80.58 & 75.03 & 56.51 & 4.0 & 2.2 & 1.82 & 6.00 & SS \\
216 & B: 1713 & Badshah Shog & 80.04 & 73.76 & 43.71 & 4.2 & 2.1 & 2.00 & 5.83 & SS \\
217 & B: 2039 & Badshah Shog & 80.87 & 75.00 & 63.23 & 4.6 & 2.3 & 2.00 & 5.17 & MS \\
218 & B: 286 & Badshah Shog & 80.51 & 71.85 & 49.26 & 5.1 & 2.0 & 2.55 & 5.67 & SS \\
254 & Bd: 105 & Badshah Shog & 79.79 & 70.39 & 63.00 & 4.1 & 2.0 & 2.05 & 5.67 & SS \\
\hline Range & & & & & & & & & \\
Min & & & 79.33 & 70.39 & 39.02 & 3.7 & 1.2 & 1.82 & 4.00 & \\
Max & & 82.31 & 80.54 & 73.69 & 6.8 & 2.3 & 3.25 & 6.33 \\
\hline
\end{tabular}

HULL\%-Hulling percentage, MILL\% -Milling percentage, HRR\%-Head Rice Recovery Percentage, KL- Kernel Length, KB- Kernel Beadth, L/B-

Kernel Length/Breadth ratio, ASV-Alkali Spreading Value. 
For Gelatinization temperature (Alkali Spreading Value) is the measure of cooking ease and is indexed by alkali digestibility test (Little et al., 1958). No entry was found low rating of alkali spreading value. Out of 32 entries 7 (B: 2094, B: 2604, B: 1307 B: 1005, B: 2302, B: 1029 and B:2321) were observed in intermediate scale value 4-5, 23 in scale value 5-6 and remaining 2 accessions (B: 2812 and B: 2504) in scale value 6-7 (Table 3 and 4). The intermediate rating (4-5) indicates medium disintegration and classified as intermediate gelatinization temperature and highly desirable for quality grain (Bansal et al., 2006).

Table 4. Top ranking accessions from predominant groups.

\begin{tabular}{|l|l|l|}
\hline \multicolumn{1}{|c|}{ Characters } & $\begin{array}{c}\text { No. of } \\
\text { accessions }\end{array}$ & \multicolumn{1}{c|}{ Best donors } \\
\hline $\begin{array}{l}\text { Top ranking } \\
\text { accessions for yield } \\
\text { (kg/ha) }\end{array}$ & 10 & $\begin{array}{l}\text { B. 2816 (4630). B: 2039 (4389) B: 2324, } \\
\text { B: 1639 (3722), B: 2094 (3685), B: 2461 } \\
\text { (3361), B: 249 (3361), B: 1727 (3296), B: } \\
\text { 169 (323)) B: 1340 (3194) }\end{array}$ \\
\hline $\begin{array}{l}\text { High Milling } \\
\text { percentage ( }>75 \%)\end{array}$ & 14 & $\begin{array}{l}\text { B: 1029, B: 799 11, B: 1322, B 2354, B: } \\
\text { 670 I, B: 1717, B: 1693, B: 2461, B: 2464, } \\
\text { B: 421, B: S28, B: 2504, B: 1639, B: 2039 }\end{array}$ \\
\hline $\begin{array}{l}\text { High Head Rice } \\
\text { Recovery } \\
\text { percentage (>65\%) }\end{array}$ & 6 & $\begin{array}{l}\text { B: 1029, B: 799 II, B: 1389, B: 528, B: } \\
\text { 2495, B: 2604 }\end{array}$ \\
\hline $\begin{array}{l}\text { Kernel length (mm) } \\
(>5.5)\end{array}$ & 2 & B: 1307, B: 2816 \\
\hline $\begin{array}{l}\text { Length/Breadth } \\
\text { ratio (upto 3) }\end{array}$ & 3 & $\begin{array}{l}\text { B: 1389 (short slender) B: 1307 (long } \\
\text { slender) B: 2816 (long slender) }\end{array}$ \\
\hline $\begin{array}{l}\text { Desirable Alkali } \\
\text { Spreading Value } \\
(4-5 \text { scale) }\end{array}$ & 7 & $\begin{array}{l}\text { B: 2321, B: 1029, B: 2302, B: 1307, B: } \\
\text { 1005, B: 2094, B: 2604 }\end{array}$ \\
\hline
\end{tabular}

From the present experiment, the desirable accessions combination of higher yield with quality parameters were found i.e 3 accessions (B: 2039, B: 2354 and B: 1639) in high yield with high head rice recovery. 5 accessions (B: 2816. B: 2039, B: 2354, B: 1639, and B: 2094) high milling \% and one (B: 094) with intermediate alkali value (Table 5) 
Table 5. Screening of accessions for desirable traits with higher yield.

\begin{tabular}{|l|l|l|}
\hline \multicolumn{1}{|c|}{ Characters } & $\begin{array}{c}\text { No. of } \\
\text { accessions }\end{array}$ & \multicolumn{1}{c|}{ Best donors } \\
\hline $\begin{array}{l}\text { High yield with Plant height (upto } \\
135 \mathrm{~cm})\end{array}$ & 1 & B: 1340 \\
\hline $\begin{array}{l}\text { High yield with long panicle length } \\
(>30 \mathrm{~cm})\end{array}$ & 2 & B: 2039, B: 2495 \\
\hline $\begin{array}{l}\text { High yield with more number of } \\
\text { effective tillers per hill ( } \geq 7)\end{array}$ & 2 & B: 2816, B: 1639 \\
\hline $\begin{array}{l}\text { High yield with High Head Rice } \\
\text { Recovery\% }\end{array}$ & 3 & B: 2039, B: 24 B: I639 \\
\hline $\begin{array}{l}\text { High yield with High Milling } \\
\%(>70 \%)\end{array}$ & 5 & $\begin{array}{l}\text { B: } 2816, \text { B: } 2039, \text { B: } \\
2354, \text { B: } 1639, \text { B: } 2094\end{array}$ \\
\hline $\begin{array}{l}\text { High yield with Desirable Alkali } \\
\text { Spreading Value (4-5) }\end{array}$ & 1 & B: 2094 \\
\hline
\end{tabular}

These indigenous aromatic rice having a lot of potential for various traits and could be used for further improvement for incorporating certain important and valuable traits. The basic study on genotypes for yield contributing characters of traditional aromatic varieties and other quality traits would help in making precised breeding strategies (Sarawgi and Bisne, 2006). These scented rice accessions B: 1340, B: 2039, B: 2495, B: 2816, B: 1693, B: 2354, B. 1639, B: 2094 may be used for different agronomical and quality traits in hybridization programme to achieve desired segregants for good grain quality with higher yield

\section{References}

Bansal U.K., H. Kaur and R.G. Saini. 2006. Donors for quality characteristics in aromatic rice. Oryza 43 (3): 197-202.

Little R.R., G.B. Hilder and E.H Dawson. 1956. Differential effect of dilute alkali on 25 varieties of milled white rice. Cereal Chem. 35 (2): 11-126.

Nayak A.R., J.N. Reddy and A.K. Pattnaik 2002. Quality evaluation of some Thailand and Vietnam scented rice. Indian J. Plant Genet. 15 (2): 125-127.

Patil P.V., A.K. Sarawgi and M.N Shriastava. 2003. Genetic analysis of yield and quality traits in traditional aromatic accessions of rice. J. Maharashtra Agric. 28 (3): 255-258

Sarawgi A.K. and Bisne Rita 2006. Genetic analysis of aroma in some aromatic cultivars of rice. J. Maharashtra Agric. Univ. 31 (3): 268-26

Saxena R.K., T.T. Chang, R.L. Sapra and R.S. Paroda 1088. Evaluation studies in indigenous rice (Oryza sativa L.) germaplasm at IRR1. Philippines. Published in NBPGR manual pp. 1-3 
Singh B.N., S.R. Dhua, R.K. Sahu, B.C. Patra and B.C. Marndi. 2001. Status of rice germplasm-Its collection and conservation in India Indian J. Plant. Genet Resour. 14: $105-106$.

Subba Rao L.V., G.S.V. Prasad, U. Prasada Rao, A. Rama Prasad, T.L. Acharyulu and S. Rama Krishna. 2001. Collection, characterization and evaluation of rice germplasm from Bastar Region. Indian J. Plant. Genet Resour. 14: 222-224. 